\title{
Autosomal Dominant Polycystic Kidney Disease With Idiopathic Membranous Nephropathy: An Unusual Association?
}

\author{
Antonio Granata ${ }^{\mathrm{a}, \mathrm{e}}$, Lidia Puzzo ${ }^{\mathrm{b}}$, Fulvio Floccaric ${ }^{\mathrm{c}}$, Carmelo Erio Fiore ${ }^{\mathrm{d}}$
}

\begin{abstract}
Autosomal dominant polycystic kidney disease (ADPKD) is usually characterized by proteinuria less than $1 \mathrm{~g} / 24$ hours and only anecdotal cases of associated nephrotic syndrome have been reported. We report another case of ADPKD with nephrotic syndrome, where histological examination showed features of membranous glomerulonephritis. Serologic tests for hepatitis B surface antigen and anti-hepatitis $\mathrm{C}$ antibodies were negative. Antinuclear antibody and anti-neutrophil cytoplasmic antibody were negative. Serum complement fraction, $\mathrm{C} 3$ and $\mathrm{C} 4$, were within normal range. Abdominal ultrasound examination showed both enlarged liver and kidneys, both with multiple cysts of varying sizes. Nevertheless renal parenchyma between cysts was not yet widely interested. Family history revealed ADPKD affecting the mother. Considering nephrotic proteinuria, a percutaneous biopsy was performed, and a diagnosis of idiopathic membranous nephropathy associated with ADPKD was made. Our patient was managed with salt restriction, diuretics and antihypertensive drugs (ACE-I and ARB) and started therapy according to Ponticelli's Protocol. At 12 week follow up proteinuria dropped to less than $1 \mathrm{~g} / 24$ hours, with normalization of renal function. Our case confirmed the importance of kidney biopsy even in patients with ADPKD and nephrotic syndrome, in order to demonstrate any coexisting glomerular disease, make an accurate diagnosis and plan appropriate treatment.
\end{abstract}

Manuscript accepted for publication February 4, 2011

\footnotetext{
aDepartment of Nephrology and Dialysis, AOU "Policlinico - Vittorio Emanuele", Catania, Italy

${ }^{\mathrm{b}}$ Department G.F. Ingrassia, Section of Pathology, University of Catania, Catania, Italy

${ }^{\mathrm{c} D e p a r t m e n t ~ o f ~ N e p h r o l o g y ~ a n d ~ D i a l y s i s, ~ O s p e d a l e ~ S a n ~ P a o l o, ~}$ Civitavecchia, Italy

${ }^{\mathrm{d}}$ Department of Internal Medicine, AOU "Policlinico - Vittorio Emanuele", Catania, Italy

${ }^{\text {e}}$ Corresponding author: Antonio Granata, Via F. Paradiso 78/a, I-95024

Acireale (Catania), Italy. Email: antonio.granata4@tin.it
}

doi:10.4021/jmc144w
Keywords: Nephrotic syndrome; Autosomal dominant polycystic kidney disease; Idiopathic membranous nephropathy; Kidney biopsy

\section{Introduction}

Autosomal dominant polycystic kidney disease (ADPKD) is usually characterized by proteinuria less than $1 \mathrm{~g} / 24$ hours and only anecdotal cases of associated nephrotic syndrome have been reported [1]. Focal segmental glomerulosclerosis, membranous nephropathy, minimal change disease, crescentic glomerulonephritis, IgA nephropathy, mesangioproliferative glomerulonephritis, diabetic glomerulosclerosis and amyloidosis were reported in these cases.

Membranous nephropathy, the most common cause of idiopathic nephrotic syndrome in adults, was reported only in three cases of ADPKD with nephrotic syndrome in literature [1-4]. It is our opinion that we do not perceive correctly epidemiology of glomerulonephritis in these patients, too rarely undergoing renal biopsy. Many nephrologists are in fact probably reluctant to plan a biopsy in polycystic patients, because of perceived increased risk of post-procedural bleeding.

\section{Case Report}

We here reported a case of ADPKD, affected by nephrotic syndrome, where histological examination showed features of membranous glomerulonephritis.

A 25-year-old man was admitted in our department for swelling of face and feet in the last one month. He denied any history of abdominal pain, fever, dysuria, smoky urine, gross hematuria, sore throat, skin infection, joint pains, skin rash, oral ulcers and using drugs; his blood pressure was 150/100 mmHg. Physical examination was unremarkable, except for bilaterally palpable knobby kidneys and edemas.

Hematological test showed: hemoglobin $141 \mathrm{~g} / \mathrm{L}$, total leukocyte count $6880 / \mathrm{mm}^{3}$ (Neutrophils $68 \%$, Lymphocytes 


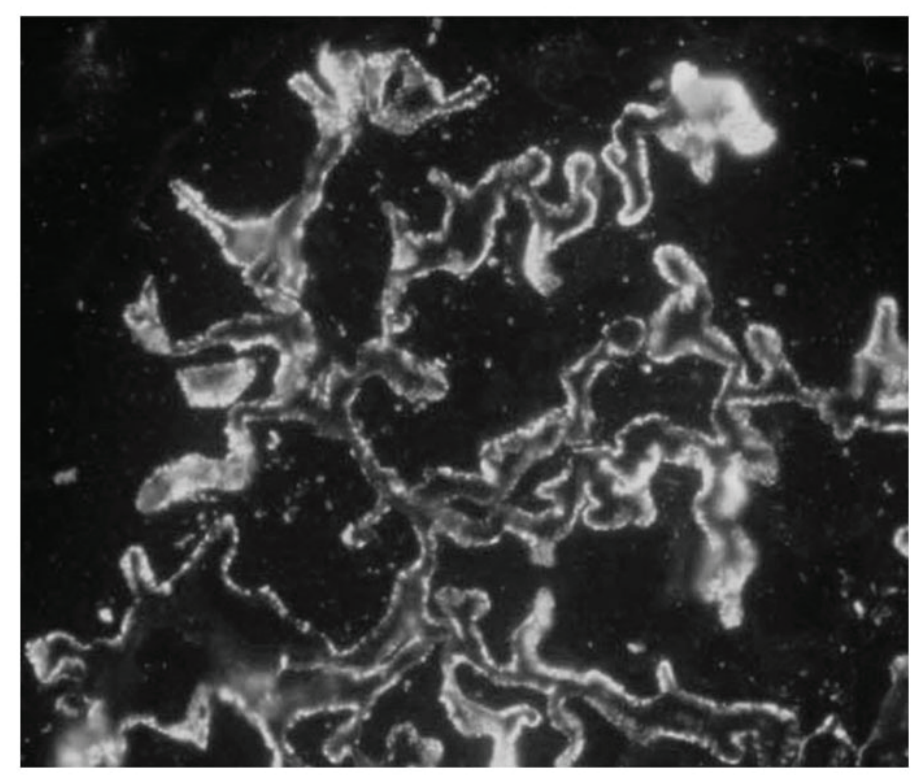

Figure 1. IgG: granular subendothelial deposits along capillary walls. (IF, $40 \mathrm{X}$ )

$27 \%$ ), platelet count $3.1 \times 10^{5} / \mathrm{mm}^{3}$, blood urea nitrogen 7.2 $\mathrm{mmol} / \mathrm{L}$, serum creatinine $179 \mu \mathrm{mol} / \mathrm{L}$, serum potassium $4.1 \mathrm{mmol} / \mathrm{L}$, serum albumin $28 \mathrm{~g} / \mathrm{L}$ and serum cholesterol $117.13 \mathrm{mmol} / \mathrm{L}$. Urine analysis showed: 4+ albumin, 10 - 15 leukocytes and $8-10$ red blood cells/HPF. Urinary protein excretion was $5.27 \mathrm{~g} / 24$ hours. Urine culture and throat swab culture were sterile.

Serologic tests for hepatitis B surface antigen and antihepatitis $\mathrm{C}$ antibodies were negative. Antinuclear antibody (ANA) and anti-neutrophil cytoplasmic antibody (ANCA) were negative. Serum complement fractions, C3 and C4, were within normal range.

Abdominal ultrasound examination showed both enlarged liver and kidneys, both with multiple cysts of varying sizes. Nevertheless renal parenchyma between cysts was not yet widely interested. Family history revealed ADPKD affecting the mother. Based on this finding, the diagnosis of ADPKD was performed.

Considering nephrotic proteinuria, a percutaneous biopsy was performed, with no reported peri-procedural complications. The specimen contained 14 mildly enlarged glomeruli, with rigid appearance of capillary wall, mild thickened basal membrane (PAS +, Congo Red -) with small spike like projections (Silver stain + ); direct immunofluorescence on cryostatic slides highlighted granular sub endothelial IgG and C3c (Fig. 1) deposits along glomerular capillary wall, while the other immunoglobulins (IgM, IgA) and complement fractions $(\mathrm{C} 4 \mathrm{c}, \mathrm{C} 1 \mathrm{q})$ were normal.
Therefore, a diagnosis of idiopathic membranous nephropathy associated with ADPKD was made.

Our patient was managed with salt restriction, diuretics and antihypertensive drugs (ACE-I and ARB) and started therapy according to Ponticelli's Protocol [5]. At 12 week follow up proteinuria dropped to less than $1 \mathrm{~g} / 24$ hours, with normalization of renal function.

\section{Discussion}

This case confirmed the importance of kidney biopsy even in patients with ADPKD and nephrotic syndrome, in order to demonstrate any coexisting glomerular disease, make an accurate diagnosis and plan appropriate treatment.

In our case, among the cysts, kidney parenchyma was preserved, so it was possible to perform a percutaneous needle biopsy instead of an open biopsy. An accurate, preliminary, ultrasonographic and color-Doppler study was performed to plan needle trajectory, in order to avoid vascular structures and cysts. Targeting a cyst-free zone of lower pole allowed us to obtain a procedure totally free of adverse events.

Membranous nephropathy is the most common cause of idiopathic nephrotic syndrome in adults $(25 \%)$, but it was reported only in few cases of ADPKD with nephrotic syndrome. Is it true that this glomerulonephritis is less frequent in ADPKD patients or this datum is a consequence of ne- 
phrologist reluctance to plan biopsies in these subjects?

It is our opinion that if major effort would be spent to make a histological diagnosis, membranous nephropathy will appear as a frequent diagnosis in nephrotic syndrome even in ADPKD population.

\section{References}

1. Contreras G, Mercado A, Pardo V, Vaamonde CA. Nephrotic syndrome in autosomal dominant polycystic kidney disease. J Am Soc Nephrol 1995;6(5):1354-1359.

2. Saxena S, Hotchandani RK, Bhuyan UN, Agarwal SK,
Tiwari SC, Dash SC. Membranous glomerulonephritis associated with autosomal dominant polycystic kidney disease. Nephron 1993;65(2):316-317.

3. Shikata K, Makino H, Ota Z. A membranous nephropathy associated with adult polycystic kidney disease. Clin Nephrol 1991;36(5):223-227.

4. Kengne-Wafo S, Massella L, Diomedi-Camassei F, Emma F. Idiopathic membranous nephropathy associated with polycystic kidney disease. Pediatr Nephrol 2010;25(5):961-963.

5. Ponticelli C, Passerini P. Management of idiopathic membranous nephropathy. Expert Opin Pharmacother 2010;11(13):2163-2175. 